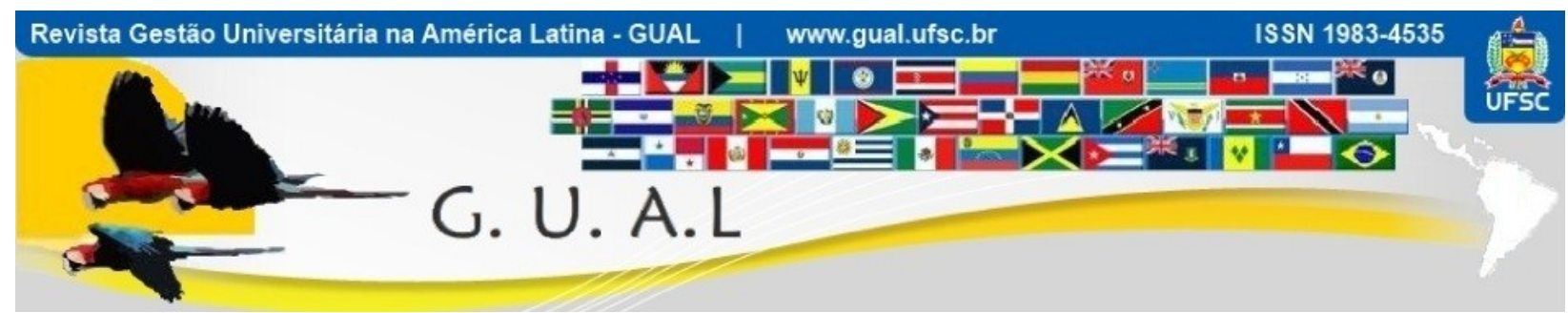

DOI: http://dx.doi.org/10.5007/1983-4535.2012v5n4p78

\title{
O USO DA GESTÃO DE PROJETOS PARA A IMPLANTAÇÃO DE UMA GESTÃO DA INFORMAÇÃO INTEGRADA E PARTICIPATIVA: A EXPERIÊNCIA DA COORDENADORIA DE EDUCAÇÃO ABERTA E A DISTÂNCIA DA UNIVERSIDADE FEDERAL DE VIÇOSA-MG
}

\author{
USE OF PROJECT MANAGEMENT FOR THE IMPLEMENTATION OF AN \\ INTEGRATED AND PARTICIPATIVE INFORMATION MANAGEMENT: THE \\ EXPERIENCE OF THE COORDINATION OF OPEN AND DISTANCE EDUCATION \\ OF FEDERAL UNIVERSITY OF VIÇOSA-MG
}

Leo Jaime Ribeiro Faria, Bacharel Universidade Federal de Viçosa - UFV leo.faria@ufv.br

Telma Regina da Costa Guimarães Barbosa, Doutora Universidade Federal de Viçosa - UFV telma@ufv.br

Recebido em 16/novembro/2012

Aprovado em 07/dezembro/2012

Sistema de Avaliação: Double Blind Review

Esta obra está sob uma Licença Creative Commons Atribuição-Uso. 


\title{
O USO DA GESTÃO DE PROJETOS PARA A IMPLANTAÇÃO DE UMA GESTÃO DA INFORMAÇÃO INTEGRADA E PARTICIPATIVA: A EXPERIÊNCIA DA COORDENADORIA DE EDUCAÇÃO ABERTA E A DISTÂNCIA DA UNIVERSIDADE FEDERAL DE VIÇOSA-MG DOI: http://dx.doi.org/10.5007/1983-4535.2012v5n4p78
}

\section{RESUMO}

A gerência da Educação a Distância exige que seus gestores executem tarefas de planejamento, sistematização, organização, direção e controle de processos e projetos e por isso não pode prescindir de um gerenciamento da informação que atenda aos requisitos de integração, de responsabilidade coletiva e de participação. Sendo assim, este trabalho teve como objetivo a implementação da gestão da informação na Coordenadoria de Educação Aberta e a Distância da Universidade Federal de Viçosa (CEAD-UFV), seguindo a metodologia de gerenciamento de projetos com base numa modificação dodotProject, um software livre de código aberto. Para tanto, foi desenvolvido e implementado um sistema de gerenciamento da informação na Coordenadoria, com base na metodologia da gestão de projetos para organizações de tecnologia da informação e comunicação. Constatou-se que o modelo implantado ofereceu à CEAD-UFV uma visão sistêmica de seus projetos e dinamizou o fluxo de informações. A experiência mostrou ainda que a gestão baseada nagerência de projetos permite enfatizar a sinergia entre grupos necessária no contexto de $\mathrm{EaD}$.

Palavras-chave: Gestão da Informação. Gestão de projetos. Educação a Distância.

\begin{abstract}
Management of Distance Education demands competences at planning, systematization, organization, and controlling of processes and projects. These tasks cannot be carried out successfully without information management system that meets the requirements of integration, collective responsibility and participation. Therefore, this study aimed at implementing an information management system at the Coordination of Open and Distance Education of Federal University of Viçosa (CEAD-UFV), following the methodology of Project Management. For this purpose, it was developed and implemented an information management system based on the methodology of project management for information, technology and communication companies, by adapting the dotProjec -, an open source software. It was noted that the model implanted offered to CEAD-UFV a systemic view of their projects and a dynamic information flow. The experience also showed that an information management based on project management allows focusing on the synergy between groups required in distance education context.
\end{abstract}

Keywords: Information Management. Project management. Distance Education 


\section{O USO DA GESTÃO DE PROJETOS PARA A IMPLANTAÇÃO DE UMA GESTÃO DA INFORMAÇÃO INTEGRADA E PARTICIPATIVA: A EXPERIÊNCIA DA COORDENADORIA DE EDUCAÇÃO ABERTA E A DISTÂNCIA DA UNIVERSIDADE FEDERAL DE VIÇOSA-MG DOI: http://dx.doi.org/10.5007/1983-4535.2012v5n4p78}

\section{INTRODUÇÃO}

A educação a distância tem se estabelecido vigorosamente. Praticamente inexistente há dez anos, correspondeu a 14,6\% do total de matrículas na graduação em 2010, a maior participação na década, de acordo com o censo da educação superior (INEP,2011). Verifica-se também o aumento da qualidade do ensino na modalidade a distância. Conforme a ABED, o resultado final dos estudantes EaD no Exame Nacional de Desempenho (ENADE) é superior ao dos estudantes de cursos presenciais. Contribui para esse quadro, de modo geral, o avanço das tecnologias de informação e comunicação e, especificamente, os esforços das universidades e faculdades em todo o território nacional e o papel fundamental de instituições tais como a UAB - Universidade Aberta do Brasil, hoje parte da CAPES, ABED e UNIREDE, entre outras.

Nas instituições federais de ensino - IFES, é nas Unidades de Educação a Distância, às vezes denominadas Núcleos ou Centros (UEaD), que esse quadro se realiza. Essas unidadesassumem duploobjetivo: desenvolver a modalidade de ensino no paíse zelar porsua qualidade, como implica o documento Referenciais de Qualidade para a Educação Superior a Distancia (BRASIL, 2007).

O modelo de Educação a Distância seguido no Brasil consiste na união de tecnologias de informação, comunicação e conteúdos instrucionais que funcionam por meio do envolvimento de estudantes, tutores, professores, instituições de ensino, órgãos do governo (MAIA, 2007), autarquias e muitos outros profissionais. Sabe-se da exigência de capital físico, estrutural e administrativo, intelectual e tecnológico, humano e financeiro, não necessariamente nesta ordem. No cumprimento da sua missão, o gestor das UEaDs deverá executar tarefas de planejamento, sistematização, organização, implementação, direção e controle de processos e projetos.

Isso equivale a dizer que esse gestor deverá planejar e organizar de forma adequada e eficiente todas as etapas do processo de criação, oferta e acompanhamento dos cursos, bem como, selecionar, dirigir, coordenar e controlar uma gama de recursos e fatores envolvidos no fluxo de atividades das UEaDs. Isso implica numa necessidade de coordenação de diferentes atividades paralelas que envolvem instituições diferentes e grupos dispersos geograficamente (AMORIM e MISKULIN, 2011). Além disso, as tarefas acima devem ser concebidas e realizadas de forma dinâmicainovadora e eficiente, no que diz respeito ao uso adequado de 


\section{O USO DA GESTÃO DE PROJETOS PARA A IMPLANTAÇÃO DE UMA GESTÃO DA INFORMAÇÃO INTEGRADA E PARTICIPATIVA: A EXPERIÊNCIA DA COORDENADORIA DE EDUCAÇÃO ABERTA E A DISTÂNCIA DA UNIVERSIDADE FEDERAL DE VIÇOSA-MG DOI: http://dx.doi.org/10.5007/1983-4535.2012v5n4p78}

recursos; estratégica e eficaz, no que diz respeito ao atendimento das necessidades da instituição e dos públicos-alvo dos projetos e cursos na modalidade a distancia.

Essas características e condições tornamcomplexo o gerenciamento das UEaDs, cuja gestão é geralmente autônoma ou no máximo mista (COSTA, 2001).Nesse contexto, as novas tecnologias de gestão ganham importância fundamental, uma vez que introduzir uma inovação ou trabalhar com tecnologias inovadoras é uma atividade que não pode ser gerida como outras atividades rotineiras de uma organização (MONTEIRO e FALSARELLA, 2006). Além disso, administrar organizações complexas significa constantemente tomar decisões e, para tanto, a informação é imprescindível. Como a informação, para ser válida, deve ser tratada, é necessário gerenciar ofluxo de informações vindo de diferentes órgãos e até mesmo regiões geográficas, priorizando sua busca, manutenção e distribuição (MONTEIRO e FALSARELLA, 2006).

Pode-se afirmar que as UEaDs não podem prescindir de um sistema de gestão de informação. A gestão da informação pode ser vista "como uma rede de processos que adquire, cria, organiza, distribui e usa a informação" (CHOO, 1998, apud PIMENTA e NETO, 2010, p. 4). No contexto da Educação a Distância o gerenciamento da informação deve atender aos requisitos de integração, da responsabilidade coletiva e de participação. Ou seja, deve integrar todas as áreas e perpassar todos os integrantes do processo bem como suas ações; deve considerar e enfatizar o fato de que as ações individuais têm impactos no resultado de todos e influencia o sistema como um todo, independentemente do setor em que se origina e deve ainda permitir e enfatizar a sinergia entre grupos (OLIVEIRA, 2006).

Sendo assim, nesse contexto a gestão de projetos ganha papel fundamental, já que segundo Monteiro e Falsarella (2006, p. 80), em “qualquer ação numa organização em termos de adaptação a mudanças ou desenvolvimento e/ou implantação deuma inovação, se faz necessário criar estruturas de projetos".

Como alternativas de ferramentas para suporte à gestão da informação com base em projetos, principalmente na área de novas tecnologias de informação e comunicação, existem diversos sistemas de gerenciamento de projetos. Entre eles está o sistema denominado dotProject que, por ser um software livre de código aberto e permitir sua customização de acordo com as necessidades da organização, torna-se vantajoso (JORDAN, 2008).

A Coordenadoria de Educação Aberta e a Distância da Universidade Federal de Viçosa (CEAD-UFV) administra vários projetos em parceria com governos estaduais e 


\section{O USO DA GESTÃO DE PROJETOS PARA A IMPLANTAÇÃO DE UMA GESTÃO DA INFORMAÇÃO INTEGRADA E PARTICIPATIVA: A EXPERIÊNCIA DA COORDENADORIA DE EDUCAÇÃO ABERTA E A DISTÂNCIA DA UNIVERSIDADE FEDERAL DE VIÇOSA-MG DOI: http://dx.doi.org/10.5007/1983-4535.2012v5n4p78}

federal, instituições parceirasdiversas, professores de vários departamentos da universidade, bolsistas e tutores dos três campi universitários. Isso confere ao fluxo de informações complexidade tal que uma estrutura organizacional tradicional não consegue gerenciar de forma eficaz. É evidente a necessidade de um sistema de gerenciamento que permita a administração das informações relativas aos projetos executados pela CEAD-UFV originadas internamente ou vindas das mais diversas fontes externas. Ou seja,um sistema que permita o gerenciamento integrado das informações entre os diversos participantes nos seus projetos.

Embora exista na instituição recursos tecnológicos e financeiros, bem como capital intelectual e humano para desenvolver um sistema próprio, tal empreendimento, além de envolver tempo,pode significar desperdício ou utilização inadequada de recursos públicos. $\mathrm{O}$ uso de um sistema livre, amplamente testado e passível de personalização, tal como o dotProject, pareceu alternativa mais efetiva e eficaz.

Levanta-se, assim, o problema desse estudo: Como implementar a gestão da informação na CEAD-UFV com base na metodologia do gerenciamento de projetos utilizando o dotProject? Assim, foi desenvolvido um trabalho visando à implementação da gestão da informação na Coordenadoria de Educação Aberta e a Distância da UFV, seguindo a metodologia de gerenciamento de projetos através do uso do softwaredotProject. E para sua concretização, buscou-se atingir os seguintes objetivos específicos: i) realizar um diagnóstico situacional da CEAD; ii) identificar e qualificar a necessidade da Gestão da Informação na Coordenadoria;iii) analisar as vantagens do Gerenciamento de Projetos na Gestão da Informação da Coordenadoria; iv) redefinir a estrutura administrativa e operacional, utilizando o gerenciamento de projetos para garantir uma eficientegestão da informação e; v) implementar um sistema de gerenciamento capaz de facilitar o fluxo e gerenciamento da informação utilizando o dotProject.

\section{FUNDAMENTAÇÃO TEÓRICA}

\subsection{GESTÃO DA INFORMAÇÃO}

A informação é o dado trabalhado que permite tomar decisões (OLIVEIRA, 1997, apud MONTEIRO e FALSARELLA, 2006). Um dado é um elemento em sua forma bruta que não possui significado próprio, mas fornece matéria prima a partir da qual é produzida a informação (ROBREDO, 2003,apudMONTEIRO e FALSARELLA,2006). 


\section{O USO DA GESTÃO DE PROJETOS PARA A IMPLANTAÇÃO DE UMA GESTÃO DA INFORMAÇÃO INTEGRADA E PARTICIPATIVA: A EXPERIÊNCIA DA COORDENADORIA DE EDUCAÇÃO ABERTA E A DISTÂNCIA DA UNIVERSIDADE FEDERAL DE VIÇOSA-MG DOI: http://dx.doi.org/10.5007/1983-4535.2012v5n4p78}

Por sua vez, a informação produz conhecimento e traz benefícios para o indivíduo e para a sociedade (BARRETO, 1994). No âmbito organizacional, Silva e Tomaél (2007) defendem que a informação é um importante ativo para o compartilhamento do conhecimento nas organizações.

A informação, como elemento que reduz a incerteza em determinada situação, é muito útil para o gerenciamento de projetos uma vez que uma das características marcantes de um projeto é a presença da incerteza quanto aos resultados a serem atingidos (MONTEIRO e FALSARELLA, 2006). Segundo os autores, conforme a ideia concebida do projeto vai se desenvolvendo, vão surgindo lacunas de informações e a partir da percepção dessas necessidades começa o processo de busca por esse insumo, depois de coletadas, as informações precisam ser selecionadas, elaboradas e analisadas para serem usadas.

Dessa forma, surge a necessidade de um sistema de gerenciamento que no caso de organizações que trabalham com projetos, precisa estar ligada à metodologia de Gestão de Projetos, visto que os níveis e características das informações mudam de acordo com as fases de execução do projeto (MONTEIRO e FALSARELLA, 2006).

Para Davenport (2002) apud Monteiro e Falsarella (2006) a gestão da informação é um conjunto estruturado de atividades que gerenciam a obtenção, distribuição e o uso da informação. Os autores defendem ainda que o gerenciamento da informação consiste no conjunto de outras atividades voltadas à informação, como o armazenamento e a retroalimentação, elemento ainda mais importante no ambiente de projetos (MONTEIRO e FALSARELLA, 2006).

Nesse sentido, é possível estabelecer uma relação direta entre informação e sua gestão em organizações baseadas em projetos. A informação é o insumo básico para a tomada de decisão em projetos, um elemento essencial em todas as fases para sua eficácia e a gestão da informação é importante nesse empreendimento porque todo projeto demanda, distribui e usa informação (MONTEIRO e FALSARELLA, 2006).

\subsection{GERENCIAMENTO DE PROJETOS}

Um projeto é um empreendimento temporário, com entradas, datas, orçamento e cronograma definidos, composto por atividades executadas sucessivamente para a criação de um produto ou serviço específico (PMI $\left.{ }^{\circledR}, 2004\right)$. 


\section{O USO DA GESTÃO DE PROJETOS PARA A IMPLANTAÇÃO DE UMA GESTÃO DA INFORMAÇÃO INTEGRADA E PARTICIPATIVA: A EXPERIÊNCIA DA COORDENADORIA DE EDUCAÇÃO ABERTA E A DISTÂNCIA DA UNIVERSIDADE FEDERAL DE VIÇOSA-MG DOI: http://dx.doi.org/10.5007/1983-4535.2012v5n4p78}

Para justificar a necessidade de um modelo de gerenciamento, um projeto precisa ser complexo e demandar sua divisão em sub-tarefas que precisam ser executadas para a concretização do seu objetivo. Além disso, a execução das sub-tarefas requer também coordenação e controles minuciosos termos de duração, precedência, custo e performance(MEREDITH e MANTEL, 1985 apud ANSELMO 2002, p. 8).

Com o aumento da complexidade dos projetos empresariais, a partir da década de 80,0 gerenciamento de projetos torna-se mais conhecido pelos administradores de grandes empresas pioneiras com estruturas produtivas baseadas em projetos (PROMO, 2008).Com a globalização, a gestão de projetos torna-se ainda mais importante e difundida, uma vez que o aumento de competitividade a tornou "uma das disciplinas que mais cresce em praticamente todas as indústrias no mundo de hoje" (RAD \& RAGHAVAN,2000 apud VIEIRA, 2002, p. $1)$.

Atualmente, além de grandes corporações e governos, micro e pequenas empresas de Tecnologia da Informação (TI) têm utilizado amplamente as técnicas e ferramentas da gestão de projetos, adaptando-as para seus projetos caracterizados pela curta duração, baixo nível estratégico e pouca utilização de recursos (VARGAS, 2005).

O desenvolvimento da gestão de projetos fez com que as instituições alterassem suas estruturas organizacionais, fazendo com que seus projetos sejam o elemento mais importante em toda a organização. Com isso, surgiu um modelo alternativo ao funcional para a estruturação de uma organização, o modelo de Estrutura por Projetos (GILDO e CLEMENTS, 2009).

Entretanto, como a mudança para uma estrutura totalmente voltada para projetos, geralmente, exige muito investimento, instituições menores e, portanto, com projetos menos complexos, optam pela Estrutura Matricial, modelo híbrido entre a estrutura funcional e a de projetos que mantém o foco no projeto, mas é capaz de manter a experiência funcional da organização (GILDO e CLEMENTS, 2009).

A partir das práticas em gerência de projetos, foram-se desenvolvendo diferentes visões sobre como os projetos devem ser gerenciados, o que fez surgir várias metodologias e técnicas para o gerenciamento de projetos (SOTILLE, 2004). Algumas das mais utilizadas são: o Project ManegementBodyofKnowledge $\left(\mathrm{PMBOK}^{\circledR}\right.$ ), o RationalUnifiedProcess (RUP ${ }^{\circledR}$ ) e o Extreme Project Manegement (XPM) (SOTILLE, 2004). 
Oguia $\mathrm{PMBOK}^{\circledR}$, um guia de boas práticas em gestão de projetos, diz que o gerenciamento de projetos é realizado através de processos que podem ser agrupados e organizados (Figura 1) com base no ciclo PDCA (plan-do-check-act). O grupo de processos de planejamento corresponde ao componente "planejar" do ciclo, enquanto o grupo de processos de execução corresponde ao "fazer" e o de monitoramento e controle ao "verificar" e “agir” (PMI®, 2004).

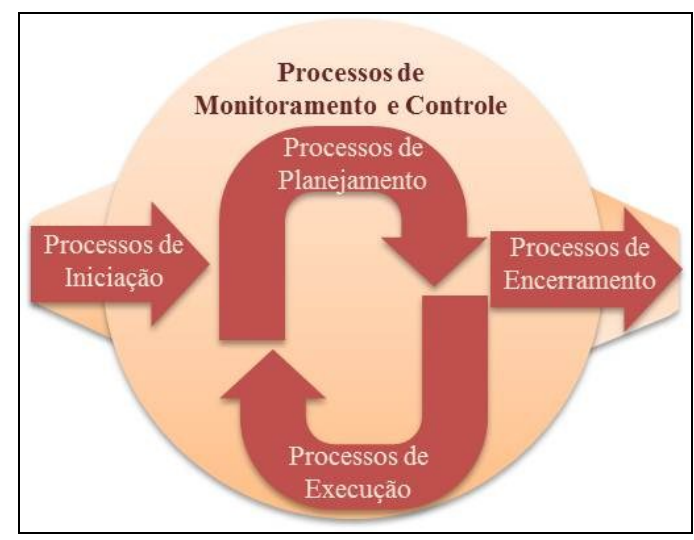

Figura 1 Grupos de Processos de Gerenciamento e o Ciclo PDCA Fonte: Adaptado de PMBOK ${ }^{\circledR}, 2004$, p. 40.

Dessa forma, o PMBOK $^{\circledR}$ oferece um conhecimento sobre a estrutura básica da Gestão de Projetos e especifica os processos de projetos utilizados pelas empresas e suas equipes para o gerenciamento de seus projetos $\left(\mathrm{PMI}^{\circledR}, 2004\right)$. Os processos definidos pelo guia se referem a aspectos da gerência de projetos e são agrupados em grandes áreas do conhecimento em gestão (SOTILLE, 2004).

\subsection{METODOLOGIA PARA A IMPLANTAÇÃO DE UM SISTEMA DE GESTÃO DA INFORMAÇÃO BASEADO NO GERENCIAMENTO DE PROJETOS}

A implantação de um sistema de gestão da informação com base no gerenciamento de projetos numa organização envolve uma série de atividades a serem implementadas em conjunto ou parcialmente, visando os objetivos sem causar muito impacto, para que possa ultrapassar as barreiras naturais de resistência às mudanças do indivíduo (ANSELMO, 2002).

Nesse sentido, o Bridges e Crawford (2000) apudAnselmo(2002) oferecem uma metodologia para implantar o gerenciamento de projetos instituindo-ocomo um projeto da organização, dividido em fases (Quadro1). 


\section{O USO DA GESTÃO DE PROJETOS PARA A IMPLANTAÇÃO DE UMA GESTÃO DA INFORMAÇÃO INTEGRADA E PARTICIPATIVA: A EXPERIÊNCIA DA COORDENADORIA DE EDUCAÇÃO ABERTA E A DISTÂNCIA DA UNIVERSIDADE FEDERAL DE VIÇOSA-MG DOI: http://dx.doi.org/10.5007/1983-4535.2012v5n4p78}

\begin{tabular}{|c|c|}
\hline Fase 1 - Fundação & $\begin{array}{l}\text { Determinam-se os interesses da organização } \\
\text { quanto à gestão de projetos e analisam-se a } \\
\text { realidade da instituição e escolha da } \\
\text { metodologia para suas características. }\end{array}$ \\
\hline Fase 2 - Iniciativas de curto prazo & $\begin{array}{l}\text { Desenvolvem-se as ferramentas e técnicas a } \\
\text { serem aplicadas na organização, bem como } \\
\text { adaptação da metodologia à realidade onde ela } \\
\text { é implementada. }\end{array}$ \\
\hline Fase 3 - Soluções de longo prazo & $\begin{array}{l}\text { Desenvolvimento de pessoas, por meio de } \\
\text { treinamento e comunicação sobre as } \\
\text { mudanças a serem realizadas, quais as } \\
\text { melhorias pretendidas e como as pessoas } \\
\text { deverão agir para uma implementação bem } \\
\text { sucedida. }\end{array}$ \\
\hline Fase 4 -Crescimento e suporte & $\begin{array}{l}\text { Nesta fase, a gestão de projetos já está } \\
\text { alinhada à estratégia da organização e a } \\
\text { preocupação passa a ser o contínuo } \\
\text { acompanhamento, avaliação e } \\
\text { desenvolvimento de acordo com o portfólio de } \\
\text { projetos. }\end{array}$ \\
\hline
\end{tabular}

Quadro 1 Fases para a implantação da Gestão de Projetos

Fonte: Elaborado a partir de Bridges e Crawford (2000) apud Anselmo (2002)

\section{METODOLOGIA}

\subsection{DELINEAMENTO DA PESQUISA E COLETA DE DADOS}

O presente trabalho se caracteriza principalmente por sua natureza qualitativa. Concorrem para essa classificação, segundo as orientações em Günther (2006), as seguintes características: seu objetivo precípuo, que é o de compreender um fenômeno social e seu significado numa situação específica; a consideração do objeto de estudo em sua forma ampla e dentro de sua historicidade; a interpretação dos resultados baseada na contextualidade e não na abstração e; finalmente, uma interação dinâmica entre o pesquisador e o seu objeto de estudo, característica mais marcante das pesquisas qualitativas.

Quanto aos fins, o estudo é descritivo uma vez que procura expor as características de determinado fenômeno, estabelecendo correlações entre variáveis e definindo sua natureza, com o objetivo de explicar ou servir de base para a explicação do fenômeno em estudo (VERGARA, 2005).

Quanto aos meios, trata-se de um estudo de caso uma vez que através dele podem-se estudar poucas unidades, com caráter de profundidade e detalhamento, o que o torna eficaz para o estudo de uma pessoa, empresa, órgão público ou comunidade (VERGARA, 2005).Além disso, o estudo de caso se enquadra nos objetivos do trabalho, uma vez que é o 


\section{O USO DA GESTÃO DE PROJETOS PARA A IMPLANTAÇÃO DE UMA GESTÃO DA INFORMAÇÃO INTEGRADA E PARTICIPATIVA: A EXPERIÊNCIA DA COORDENADORIA DE EDUCAÇÃO ABERTA E A DISTÂNCIA DA UNIVERSIDADE FEDERAL DE VIÇOSA-MG DOI: http://dx.doi.org/10.5007/1983-4535.2012v5n4p78}

mais indicado para pesquisas organizacionais. Trata-se de um método com foco na ampliação da experiência e compreensão do fenômeno estudado, sem a intenção de generalizar seus resultados (CESAR, 2005).

Por se tratar de estudo qualitativo, os métodos e ferramentas indicados são a entrevista, levantamento de dados em documentos e a observação participante (GIL 1995). O autor descreve a entrevista como uma técnica em que o pesquisador apresenta-se ao investigado e lhe formula perguntas para obter dados de interesse. Já a observação participante pode ser utilizada como procedimento científico, á medida que serve a um objetivo formulado de investigação, apresentando como principal vantagem a obtenção direta dos dados, sem intermediação.

\subsection{METODOLOGIA PARA A IMPLANTAÇÃO DA GESTÃO DE PROJETOS NA CEAD-UFV}

A principal metodologia seguida para desenvolver a gestão de projetos na CEAD-UFV foi o guia $\mathrm{PMBOK}^{\circledR}$. O sistema de gerenciamento foi então instituído a partir da elaboração de um cronograma de atividades organizadas quinzenalmente em quatro fases.

$\mathrm{Na}$ Fase I foi realizado um diagnóstico sobre as condições da CEAD-UFV quanto aos fluxos de informação e necessidade de um sistema de gerenciamento dainformação baseado na gestão de projetos. Ao final desta fase, obteve-se a adequação da metodologia escolhida à realidade do órgão e a reestruturação administrativa conforme os objetivos institucionais e da gestão de projetos para a administração eficaz das informações.

A Fase II constituiu-se na configuração e desenvolvimento de um sistema de gerenciamento de projetos,com base no software livre dotProject, que permitisse gerir as informações sobre os projetos executados pelo órgão, de acordo com as características e necessidades identificadas na CEAD-UFV.

A Fase III iniciou-se com a escolha de um setor da CEAD-UFV para a implantação do sistema de gerenciamento. O setor escolhido foi o de Produção Audiovisual e Design, dado a característica de curto prazo de seus projetos, o que permitiria uma melhor avaliação do sistema implantado num menor período de tempo.

O primeiro passo para a realização desta fase foi a apresentação do sistema aos colaboradores do setor, seguido de um treinamento individual sobre como utilizar o software. Em seguida, houve o acompanhamento e avaliação do sistema implantado com base na 
observação e questionamentos junto aos usuários. Por fim, realizaram-se as alterações no sistema de acordo com os dados coletados pela avaliação.

A Fase IV, última etapa da implementação, consistiu principalmente na aplicação do sistema para outros setores da CEAD, seguida do acompanhamento, avaliação e melhoria do mesmo.

\subsection{O SOFTWARE UTILIZADO}

Originalmente chamado de DotMarketing pelos seus criadores no ano 2000, odotProject (Figura 2) é um software livre de gerenciamento de projetos, com um conjunto de funcionalidades e características que atendem às necessidades de um sistema de gerenciamento de projetos numa organização (JORDAN, 2008).

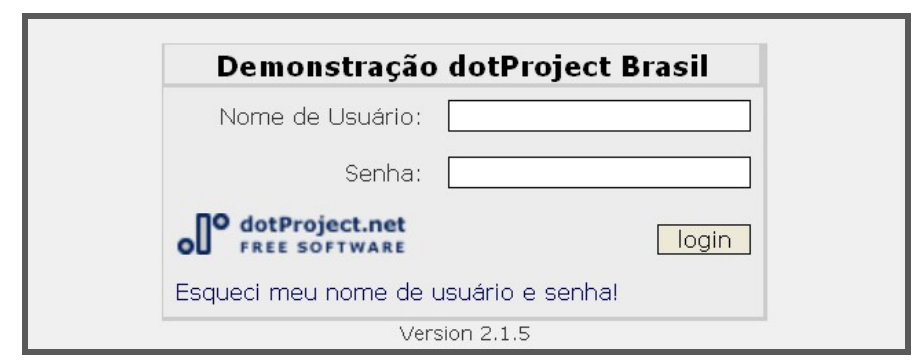

Figura 2 Tela inicial do DotProject

Fonte:http://demo.dotproject.com.br/

Tendo como principal objetivo ser uma alternativa aos softwares proprietários de gerenciamento da "Arte do Projeto", ele continua sendo desenvolvido pela mesma equipe que o criou e mais uma comunidade de voluntários de todo o mundo, além dos próprios usuários que o descobrem, aperfeiçoam e compartilham suas experiências (DOTPROJECT, 2007).

Além de ser livre e de código aberto, o que permite sua customização de acordo com as características da organização, seu acesso é feito através de um navegador web, o que torna sua utilização independente de sistema operacional ou instalação na máquina do usuário, além das informações relativas aos projetos poderem ser acessadas de qualquer local (JORDAN, 2008). Tais características foram determinantes para sua escolha como software para a gestão de projetos na CEAD-UFV.

\section{APRESENTAÇÃO E ANÁLISE DOS RESULTADOS}

\subsection{FASE I: DIAGNÓSTICO E PLANEJAMENTO}




\subsection{A SITUAÇÃO ATUAL E NECESSIDADE DA GESTÃO DA INFORMAÇÃO NA} CEAD-UFV

A Coordenadoria de EAD da UFV foi instituída em 2001 e é responsável pela coordenação, assessoramento, supervisão e suporte técnico para a utilização de Novas Tecnologias da Informação (NTICs) em diferentes áreas do ensino. Vinculada à reitoria da UFV, é regida pelas determinações da Resolução 06/2007 do Conselho Universitário.

A CEAD-UFV é responsável pela promoção e produção de 2 (dois) cursos de licenciatura, 5 (cinco) cursos de pós-graduação lato-sensu, 3 (três) cursos técnicos, 7 (sete) de capacitação profissional, todos na modalidade a distância, além de produzir materiais didáticos com a utilização de novas tecnologias para 54 (cinquenta e quatro) disciplinas presenciais da UFV. Além disso, o órgão é responsável pelo desenvolvimento e manutenção de 4 (quatro) portais e 1 (uma) série didática onlineque tem como objetivo compartilhar o conhecimento para a comunidade acadêmica e sociedade em geral.

Embora não exista uma estrutura formal administrativa que englobe todos os colaboradores e as atividades de cada setor sejam executadas independentemente, a CEAD possui uma estrutura informal bem parecida com um organograma funcional, em que as relações entre os colaboradores são regidas por seus cargos e funções (Figura 3).

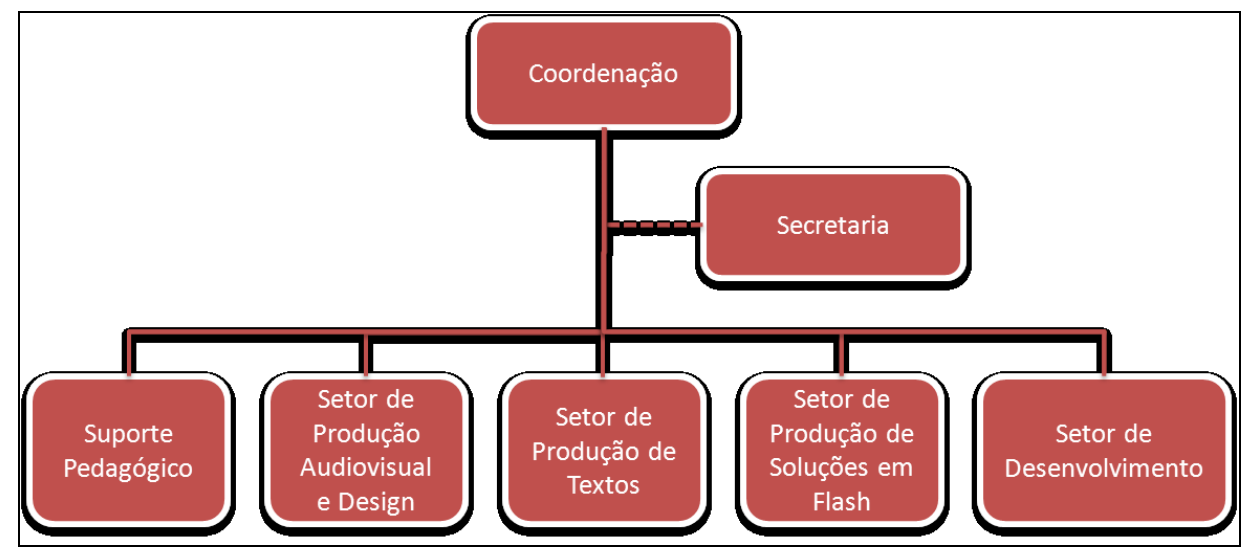

Figura 3 Organograma funcional informal da CEAD-UFV Fonte: Elaborado pelos autores

Essa estrutura leva a uma desvantagem: as informações ficamretidas em seus setores e não são compartilhadas de forma integrada pelas demais áreas do órgão. Assim, os setores tendem a trabalhar de forma isolada e os coordenadores de um setor não têm acesso direto às informações do outro. Com isso, o fluxo de informações possui muitos intermediários, uma 


\section{O USO DA GESTÃO DE PROJETOS PARA A IMPLANTAÇÃO DE UMA GESTÃO DA INFORMAÇÃO INTEGRADA E PARTICIPATIVA: A EXPERIÊNCIA DA COORDENADORIA DE EDUCAÇÃO ABERTA E A DISTÂNCIA DA UNIVERSIDADE FEDERAL DE VIÇOSA-MG DOI: http://dx.doi.org/10.5007/1983-4535.2012v5n4p78}

vez que o conhecimento ou está restrito a um setor, ou é compartilhado apenas pela coordenação do órgão.

A disseminação da $\mathrm{EaD}$, aliado ao desenvolvimento das Tecnologias da Informação e concentração das atividades voltadas para o ensino a distância na CEAD-UFV, tornou a estrutura funcional do órgão precária para a exigência cada vez mais acentuadade integração e dinamismo das informações relacionadas aos projetos executados pelo órgão.

Além disso, os projetos geralmente integram mais de um setor da CEAD-UFV, com órgãos externos parceiros, profissionais que não possuem cargos ligados à instituição, professores e tutores de diversos departamentos e comunidade de estudantes em campi universitários distantes do campus sede. Isso confere ao fluxo de informações uma complexidade que a estrutura organizacional vigente não consegue gerenciar de forma eficaz.

As principais consequências da falta de um sistema de gerenciamento integrado e de acordo com as características do órgão são o desconhecimento pelos participantes do projeto como um todo, o não conhecimento da situação de um projeto executado no órgão, incapacidade de determinar prioridades e altos custos com retrabalho devido à não difusão da informação sobre atividades desenvolvidas para todos os interessados.

Tomando conhecimento dessa realidade, percebeu-se que uma metodologia de gerenciamento orientada a projetos seria útil para a CEAD-UFV integrar o fluxo de informações e torná-las acessíveis a todos os stakeholders de seus projetos, além de permitir o acompanhamento em tempo real das atividades aumentando, contudo a qualidade dos seus resultados no que consiste à concretização de seus objetivos organizacionais.

\subsection{O FLUXO DE INFORMAÇÕES NA CEAD-UFV E AS VANTAGENS DO GERENCIAMENTO DE PROJETOS PARA A SUA GESTÃO}

Após analisar como são executadas as atividades operacionais e administrativas do órgão, observou-se que as mesmas estão diretamente relacionadas aos seus projetos, sendo o compartilhamento das informações sobre seu andamento com todos os interessados no projeto ao qual fazem parte muito importante, inclusive para a concretização dos objetivos de tais projetos.

O estudo dos fluxos de informações mostrou que os projetos são executados por meio de processos delegados aos diferentes setores do órgão, além dos projetos exigirem uma 


\section{O USO DA GESTÃO DE PROJETOS PARA A IMPLANTAÇÃO DE UMA GESTÃO DA INFORMAÇÃO INTEGRADA E PARTICIPATIVA: A EXPERIÊNCIA DA COORDENADORIA DE EDUCAÇÃO ABERTA E A DISTÂNCIA DA UNIVERSIDADE FEDERAL DE VIÇOSA-MG DOI: http://dx.doi.org/10.5007/1983-4535.2012v5n4p78}

interação constante com professores, profissionais e instituições externas que também precisam conhecer o seu andamento.

Sendo assim, a organização das informações a partir de uma metodologia orientada a projetos seria a mais indicada às características do órgão e aos objetivos do gerenciamento de projetos que, dentre outros, procura gerenciar as informações sobre cada etapa de um projeto e permitir seu acompanhamento em tempo real.

\subsection{REDEFINIÇÃO DA ESTRUTURA ADMINISTRATIVA COM BASE NO GERENCIAMENTO DE PROJETOS}

Para melhorar a fluidez da informação no órgão, percebeu-se a necessidade de mudar a estrutura organizacional funcional da CEAD-UFV para um modelo mais dinâmico, optando pela estrutura matricial (Figura 4), uma vez que a mesma oferece um organograma compatível com a gestão orientada a projetos, sem realizar uma profunda mudança de paradigma quanto à experiência mais funcional até então predominante. Desse modo, as relações entre os setores passaram a privilegiar o gerenciamento integrado das informações para a eficácia nos resultados dos projetos.

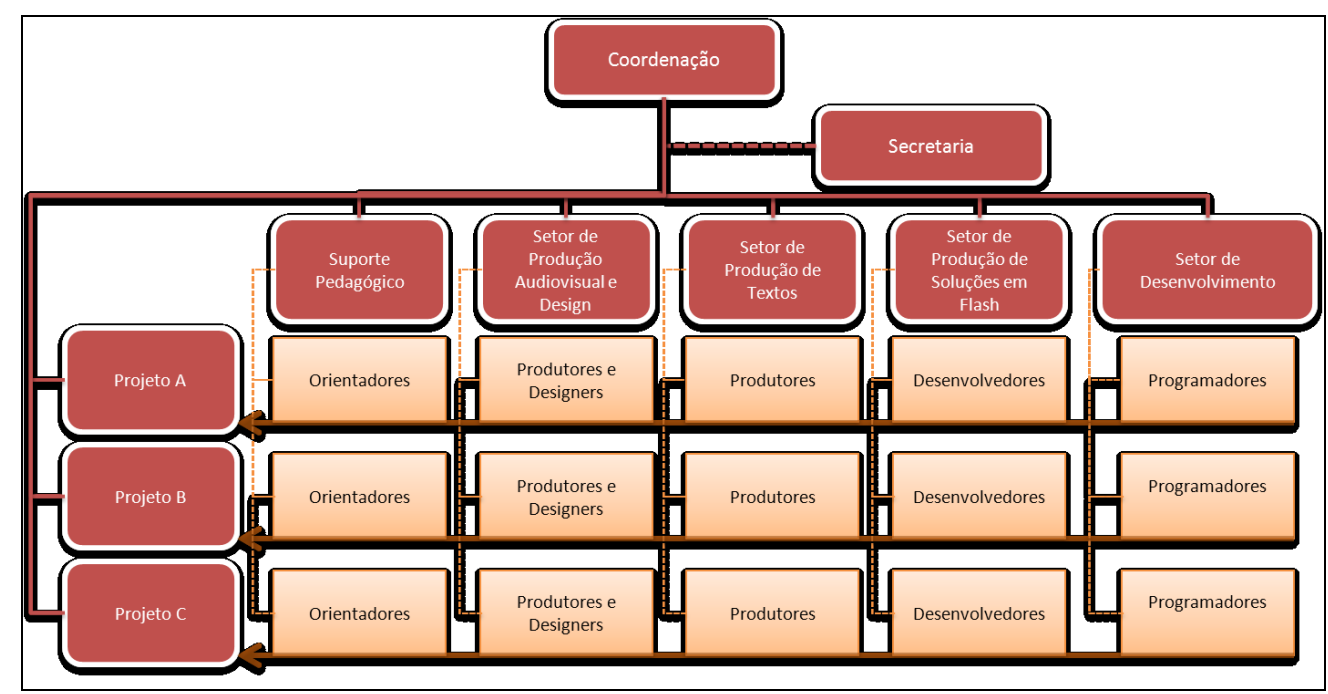

Figura 4 Organograma matricial da CEAD

Fonte: Elaborado pelos autores

Com a definição de uma estrutura matricial consegue-se manter os departamentos funcionais da antiga estrutura e a hierarquia já existente, porém integrando as relações e fluxo de informações entre os setores de acordo seu envolvimento nos projetos. Além disso, os 


\section{O USO DA GESTÃO DE PROJETOS PARA A IMPLANTAÇÃO DE UMA GESTÃO DA INFORMAÇÃO INTEGRADA E PARTICIPATIVA: A EXPERIÊNCIA DA COORDENADORIA DE EDUCAÇÃO ABERTA E A DISTÂNCIA DA UNIVERSIDADE FEDERAL DE VIÇOSA-MG DOI: http://dx.doi.org/10.5007/1983-4535.2012v5n4p78}

colaboradores continuam exercendo seus papéis funcionais na instituição, mas são alocados aos projetos de acordo com a necessidade e conveniência.

Cada projeto possui um coordenador ou uma equipe responsável pelo seu andamento. Para evitar conflitos de hierarquia, uma vez que os colaboradores se reportam aos superiores funcionais, mas também ao responsável pelo projeto, integra-se todo o processo de comunicação sobre os objetivos da CEAD-UFV e de seus projetos. Além disso, a coordenação funcional e do projeto trabalharão sempre juntas na determinação de prioridades e resolução de eventuais conflitos.

\subsection{FASE II: INSTALAÇÃO E ADAPTAÇÃO DO SOFTWARE}

A versão 2.5 do dotProject foi instalada em um servidor da CEAD-UFV utilizando a arquitetura WAMP (Windows ${ }^{\circledR}$, Apache, MySQL e PHP). Foi escolhido o sistema operacional Windows $^{\circledR}$ pelo fato da CEAD-UFV já possuir um servidor com esse sistema, o servidor web Apache pelo alto grau de utilização e sua completa compatibilidade com o software, o sistema de banco de dados $M y S Q L$ também por sua ampla utilização e não restrição em relação ao programa, e o PHP, por ser a linguagem utilizada originalmente pelo software e por sua grande difusão e fácil aprendizado, o que torna as mudanças no sistema mais simples.

Após a instalação, foi realizada a customização do sistema, retirando alguns módulos considerados desnecessários para a realidade da CEAD-UFV e acrescentando alguns para que o software correspondesse às necessidades do órgão quanto ao gerenciamento de informações.

Nesse sentido, foram identificados os perfis de usuários para o sistema de acordo com sua participação nos projetos como: i) Coordenador Geral, para o diretor da CEAD-UFV; ii) Coordenador de Setor, Coordenador de Projeto e iii) Colaborador para os usuários internos e i) Coordenador de Curso, ii) Coordenador de Disciplinas, iii) Professor e iv) Visitante, além do perfil de Administrador a ser utilizado pelo responsável pelo desenvolvimento e manutenção do sistema.

Na Página Inicial (Figura 5), além de visualizar a sua agenda e tarefas do dia ou semana, organizadas de acordo com as datas e prazos de encerramentos e projeto ao qual pertencem, acrescentou-se a opção de ver as atividades de todos os usuários e todos os projetos para incentivar uma visão sistêmica sobre os projetos e os objetivos da CEAD-UFV. 


\section{O USO DA GESTÃO DE PROJETOS PARA A IMPLANTAÇÃO DE UMA GESTÃO DA INFORMAÇÃO INTEGRADA E PARTICIPATIVA: A EXPERIÊNCIA DA COORDENADORIA DE EDUCAÇÃO ABERTA E A DISTÂNCIA DA UNIVERSIDADE FEDERAL DE VIÇOSA-MG DOI: http://dx.doi.org/10.5007/1983-4535.2012v5n4p78}

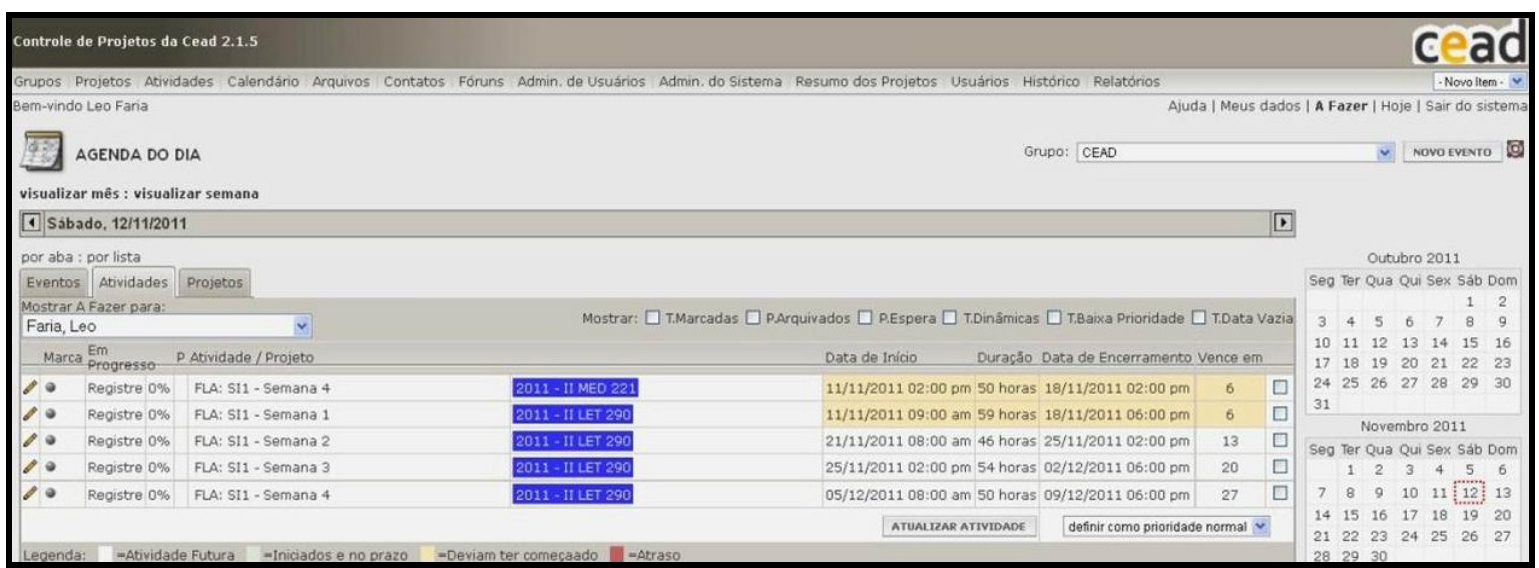

Figura 5 Página Inicial Modificada do dotProject Fonte:elaborado pelos autores

O Módulo originalmente chamado "Empresas" teve seu nome modificado para "Grupos", um termo mais adequado para a realidade da CEAD. Além do nome do módulo, outros termos do sistema foram alterados e os grupos passaram a ser usados para organizar os projetos de acordo com suas finalidades em: Novas Tecnologias da Informação (NTICs), Graduação a Distância, Mestrado Lato Sensu, Mestrado Stricto Sensu, Cursos de Curta Duração, Interno e Outros (Figura 6).

\begin{tabular}{|c|c|c|c|c|c|c|c|c|c|}
\hline \multirow{2}{*}{\multicolumn{2}{|c|}{ por aba : por lista }} & \multicolumn{2}{|c|}{$\begin{array}{l}\text { Pesquisa } \\
\text { Reiniciar Pesquisa }\end{array}$} & \multicolumn{2}{|c|}{ Filtro de Responsável } & \multicolumn{2}{|l|}{ Todos } & \multirow[t]{2}{*}{$\checkmark$} & \multirow{2}{*}{ NOVO GRUPO } \\
\hline & & & & & & & & & \\
\hline Todos os Grupos & NTICS & Graduação a Distância & Mestrado Lato Sensu & Stricto Sensu & Cursos de & Curta Duração & Interno & Outros & \\
\hline \multirow[t]{5}{*}{ classificar por: } & \multicolumn{3}{|c|}{ Nome do Grupo } & \multicolumn{2}{|c|}{ Projetos Ativos } & \multicolumn{2}{|c|}{ Projetos Arquivados } & Тіро & \\
\hline & \multicolumn{3}{|l|}{ CEAD } & & 19 & \multicolumn{2}{|c|}{15} & & Interno \\
\hline & \multicolumn{3}{|c|}{ Curso de Licenciatura em História a Distância } & & 9 & \multicolumn{2}{|c|}{0} & Gradu & uação a Distância \\
\hline & \multicolumn{3}{|c|}{ Curso de Licenciatura em Matemática a Distância } & & 4 & \multicolumn{2}{|c|}{0} & Gradu & uação a Distância \\
\hline & \multicolumn{3}{|l|}{ NTICS } & & 42 & \multicolumn{2}{|c|}{0} & & NTICS \\
\hline
\end{tabular}

Figura 6 Módulo Modificado Grupos

Fonte: elaborado pelos autores

No módulo "Projetos" (Figura 7) os projetos passaram a ser classificados de acordo com sua situação em: Indefinido, Proposto, Planejamento, Execução, Aguardando, Completo, Modelo, Arquivado e Outros. Além disso, os projetos possuem cores que os classificam de acordo com características semelhantes, tornando seu acompanhamento mais fácil principalmente para o diretor da CEAD-UFV, que coordena todos os projetos. 


\section{O USO DA GESTÃO DE PROJETOS PARA A IMPLANTAÇÃO DE UMA GESTÃO DA INFORMAÇÃO INTEGRADA E PARTICIPATIVA: A EXPERIÊNCIA DA COORDENADORIA DE EDUCAÇÃO ABERTA E A DISTÂNCIA DA UNIVERSIDADE FEDERAL DE VIÇOSA-MG DOI: http://dx.doi.org/10.5007/1983-4535.2012v5n4p78}

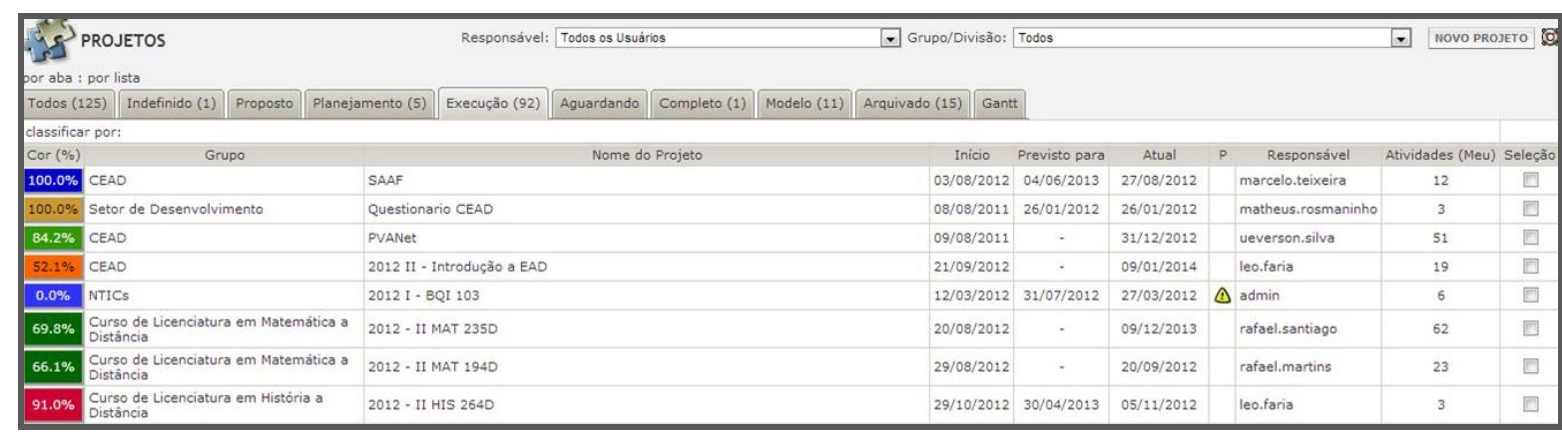

Figura 7 Módulo Modificado Projetos

Fonte: elaborado pelos autores

Por fim, no módulo "Projetos", qualquer usuário do sistema, respeitando suas permissões, obtêm informações relativas à classificação do projeto, situação, data de início e fim previstos, professor responsável pelo projeto e se há algum problema que possa atrasá-lo.

O módulo "Atividades" (Figura 8) permite aos usuários a visualização de atividades particulares, relacionadas ao seu projeto, ou todas as atividades finalizadas ou a serem realizadas na coordenadoria. As atividades de um projeto são agrupadas em Fases que identificam o material a ser produzido e, sendo assim, é possível identificar a qual setor elas pertencem.

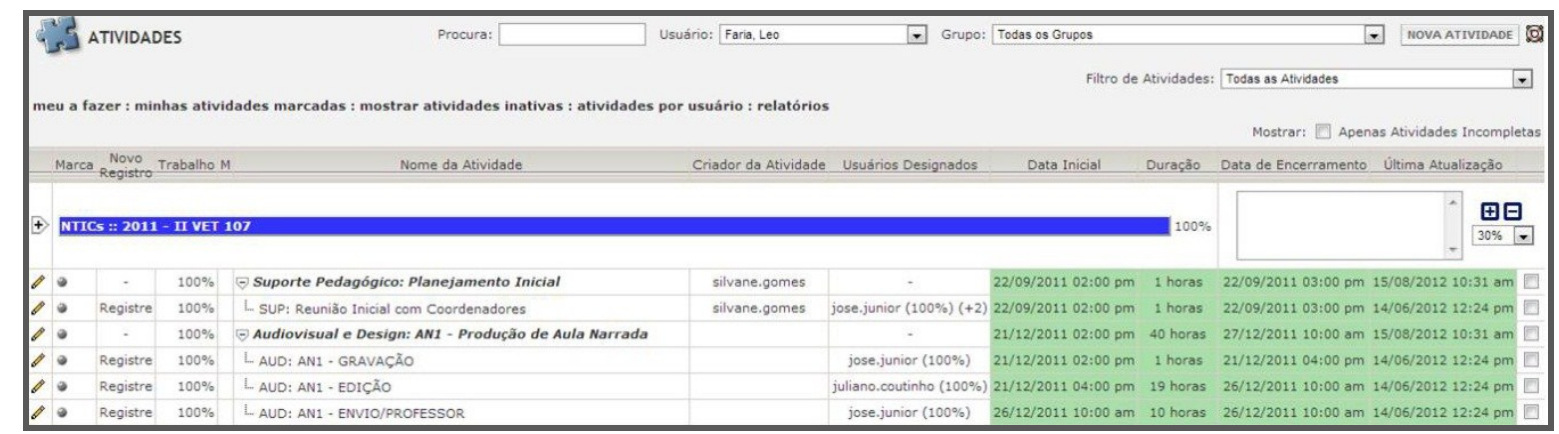

Figura 8 Módulo Modificado Atividades

Fonte: elaborado pelos autores

Além das modificações nos módulos originais do software, foram adicionados alguns módulos complementares desenvolvidos por voluntários e entusiastas do dotProject e customizados de acordo com as necessidades da CEAD-UFV.

O primeiro módulo adicionado foi o "Project Designer" que teve seu nome alterado para "Resumo dos Projetos". Esse novo recurso serve principalmente para obter informações gerais sobre o andamento de cada projeto e a possibilidade de imprimi-lo num formato melhor adaptado para sua visualização como um todo. 


\section{O USO DA GESTÃO DE PROJETOS PARA A IMPLANTAÇÃO DE UMA GESTÃO DA INFORMAÇÃO INTEGRADA E PARTICIPATIVA: A EXPERIÊNCIA DA COORDENADORIA DE EDUCAÇÃO ABERTA E A DISTÂNCIA DA UNIVERSIDADE FEDERAL DE VIÇOSA-MG DOI: http://dx.doi.org/10.5007/1983-4535.2012v5n4p78}

O segundo adicionado foi o módulo "Relatórios" que permite a produção de relatórios das informações sobre todos os projetos da CEAD-UFV de acordo com o enfoque desejado. Os relatórios podem ser mais simples para acompanhar o andamento de um projeto, ou mais complexos, com informações e índices de resultados que permitem a análise dos projetos do órgão e seus resultados como um todo.

\subsection{FASE III: IMPLEMENTAÇÃO EM UM SETOR DA CEAD-UFV}

A principal mudança decorrida desta fase de teste foi a inclusão de algumas funcionalidade no sistema de gerenciamento para facilitar a gestão de projetos e tornar o fluxo de informações mais dinâmico.

Uma importante modificação foi realizada para melhorar o fluxo de trabalho. $\mathrm{Na}$ versão original do software todas as atividades tinham datas determinadas durante o planejamento do projeto, isso fazia com que, caso uma atividade terminasse mais cedo, o colaborador seguinte só começaria sua atividade conforme programado, perdendo o tempo ganho. Sendo assim, as atividades passaram a ser interligadas, quando uma atividade é completa o sucessor é informado automaticamente pelo sistema e pode decidir se inicia ou não seu trabalho no projeto mais cedo que o programado.

POR fim, foi realizada uma avaliação com os participantes desta etapa para identificar os pontos positivos e negativos do sistema a fim de melhorá-lo e ampliá-lo para os outros setores da CEAD-UFV. Dentre os pontos positivos apontados pelos entrevistados, os que mais se destacaram foram a possibilidade de organizar formalmente os projetos e gerenciar suas informações; padronização e profissionalização na gestão, com aumento da eficiência da organização e prevenção de problemas e; possibilidade de aperfeiçoamento do sistema em si. Por outro lado, os pontos negativos foram: $O$ risco de engessamento da máquina administrativa e operacional; complexidade de uso e tempo de adaptação e; a incapacidade de lidar eficientemente com imprevistos.

Ao término desta fase, o sistema contemplava 23 projetos do Setor de Produção Audiovisual e Design da CEAD-UFV. O software já era responsável pelo controle de todas as informações e operações do setor e utilizado como principal ferramenta para gerenciamento da informação e comunicação de seus projetos. 


\section{O USO DA GESTÃO DE PROJETOS PARA A IMPLANTAÇÃO DE UMA GESTÃO DA INFORMAÇÃO INTEGRADA E PARTICIPATIVA: A EXPERIÊNCIA DA COORDENADORIA DE EDUCAÇÃO ABERTA E A DISTÂNCIA DA UNIVERSIDADE FEDERAL DE VIÇOSA-MG DOI: http://dx.doi.org/10.5007/1983-4535.2012v5n4p78}

\subsection{FASE IV: APLICAÇÃO PARA OS OUTROS SETORES}

A partir da análise das percepções e sugestões obtidas na terceira fase junto aos colaboradores do Setor de Produção Audiovisual e Design, o sistema foi rediscutido e melhorado para sua aplicação no órgão como um todo.

Para evitar uma das principais disfunções da burocracia apontada pelos entrevistados, procurou-se desenvolver um sistema totalmente online, sem a necessidade de muita documentação e processos rígidos, onde as informações podem fluir livremente e serem controladas pelo próprio software ou com o auxílio de ferramentas de apoio como sistemas de e-mail e de armazenamento e compartilhamento de arquivos online. Além disso, o sistema foi adaptado para considerar mudanças imprevistas nos prazos, uma vez que os projetos envolvem, principalmente, professores e participantes externos ao órgão.

Como não havia um sistema formal de gestão instituído na CEAD-UFV antes da implantação do dotProject, não houve muita resistência quanto ao seu uso, visto que procurou-se não mudar drasticamente as relações organizacionais, mas formalizar e adaptar o sistema de gerenciamento de informações à elas.

Portanto, o sistema é responsável pela gerência de quase a totalidade dos projetos executados pelo órgão ao final desta fase, contando com 90 projetos e 1.307 atividades cadastradas, sendo que $54 \%$ delas já se completaram.

\subsection{OS BENEFÍCIOS DO GERENCIAMENTO DE PROJETOS PARA A CEAD-UFV}

A implementação de um sistema de gerenciamento permitiu ao órgão uma administração eficaz sobre seus projetos. A partir dele, tornou-se possível medir a eficiência dos projetos e colaboradores, bem como determinar prioridades e focar no cumprimento de projetos chaves para a organização.

Outro benefício trazido pelo acesso fácil e rápido de qualquer participante às informações precisas sobre o andamento de seu projeto foi o ganho em eficiência na execução das tarefas. A partir do sistema, os colaboradores também se tornaram mais independentes, com mais autonomia para gerenciar seu próprio trabalho uma vez que puderam entender melhor suas responsabilidades nos projetos.

Além disso, foi possível desenvolver uma visão sistêmica nos colaboradores uma vez que o sistema foi planejado de modo a mostrar cada atividade ou etapa enfatizando a sua 
posição e importância no projeto como um todo e o alinhamento deste com a estratégia da instituição.

O sistema implantado, por fim, permitiu a integração entre todas as etapas dos projetos, uma vez que as informações passaram a ser tratadas e distribuídas de forma organizada para todos os interessados, criando uma comunicação eficaz entre colaboradores, coordenadores e patrocinadores dos projetos.

\section{CONCLUSÃO}

O novo modelo de gestão implantado na CEAD-UFV deu ao órgão uma visão sistêmica de seus projetos, tornou o fluxo de informações dinâmico e formalizou as relações internas e do órgão com seus parceiros. Isso colaborou para possibilitar um gerenciamento eficaz de seus projetos. Tal fato pode ser comprovado pelos relatos dos próprios colaboradores, os quais disseram que o sistema ofereceu "maior profissionalização da gestão no órgão e aumento da eficiência da organização e prevenção de problemas".

Finalmente, a experiência da CEAD-UFV serviu para mostrar que um modelo de gestão da informação baseado em projetos contribui para alcançar as características de um bom gerenciamento da informação para o contexto de $\mathrm{EaD}$, o qualpermite integrar todas as áreas e perpassar todos os integrantes do processo bem como suas ações; assim como considerar e enfatizar o fato de que as ações individuais têm impactos no resultado de todos e influencia o sistema como um todo, independentemente do setor em que se origina e ainda permite e enfatiza a sinergia entre grupos.

\section{REFERÊNCIAS}

AMORIM, J. A.; MISKULIN, R. G. S. Planejamento Estratégico para o Gerenciamento de Projetos de EaD. In: Congresso Internacional de Educação a Distância, 17, 2011, Manaus. Anais eletrônicos... Manaus: ABED, 2011. Disponível em: $<$ http://www.abed.org.br/congresso2011/cd/8.pdf>. Acesso em: 27 Set. 2012.

ANSELMO, J. L. Escritório de Gerenciamento de Projetos: Um Estudo de Caso. 2002. 89 f. Trabalho de Conclusão de Curso (Bacharelado em Administração de Empresas) Faculdade de Economia, Administração e Ciências Contábeis da Universidade de São Paulo, São Paulo, 2002.

BARreto, A. A. A Questão da Informação. Revista São Paulo em Perspectiva. São Paulo, v. 8, n 4, 1994. Disponível em: <http://aldoibct.bighost.com.br/quest/quest2.pdf $>$. Acesso em: 16 Set. 2012. 
BRASIL. Referências de Qualidade para Educação Superior a Distância. Brasília: Ministério da Educação - Secretaria de Educação a Distância, 2007.

CESAR, A. M. R. V. C. Método do Estudo de Caso (Case Studies) ou Método do Caso (Teaching Cases)? Uma análise dos dois métodos no ensino e pesquisa em Administração. REMAC: Revista Eletrônica Mackenzie de Casos. São Paulo, 2005. Disponível em: $<$ http://www.mackenzie.br/fileadmin/Graduacao/CCSA/remac/jul_dez_05/06.pdf $>$. Acesso em: 16 Nov. 2011.

COSTA, M. G. P. Ecologia da Escola: Capacitação de professores por meio da educação a distância. Educação em Foco. Belo Horizonte: FAE/CBH/UEMG, n. 5, p. 37-52, 2001. DOTPROJECT. Doc Site. Disponível em: $<$ http://docs.dotproject.net/index.php?title=Main_Page>. Acesso em: 29 Nov. 2011.

GIDO, J.; CLEMENTS, J. P. Gestão de Projetos: Tradução da $3^{\text {a }}$ Ed. Norte-americana. São Paulo: Cengage Learning, 2009. 472 p.

GIL, A. C. Métodos e Técnicas de Pesquisa Social. 4 ed. São Paulo: Editora Atlas, 1995. $207 \mathrm{p}$.

GÜNTHER, H. Pesquisa qualitativa versus pesquisa quantitativa: esta é a questão? Psicologia: Teoria e Pesquisa. Brasília, v. 22 n. 2, p. 201-210, Mai/Ago 2006.

HELDMAN, K. Gerência de Projetos: Guia para o exame oficial do PMI. 3. ed. Rio de Janeiro: Elsevier, 2006. 580 p.

INEP. Censo da Educação Superior 2010. Disponível em:

$<$ http://download.inep.gov.br/educacao_superior/censo_superior/documentos/2010/divulgaca o_censo_2010.pdf $>$. Acesso em: 20 Set. 2012.

JORDAN, L. Gerenciamento de Projetos com dotProject. São Paulo: Pearson/ Prentice Hall, 2008. 224 p.

MAIA, M. de C. Educação a Distância. GV Executivo. São Paulo: FGV-EAESP, n 5, p 5670, 2007. Disponível em: <http://rae.fgv.br/sites/rae.fgv.br/files/artigos/5017.pdf $>$. Acesso em 18 de Set. de 2012.

MONTEIRO, N. A.; FALSARELLA, O. M. Gestão da Informação em Projetos Empresariais. eGesta. Santos, v. 2, n. 1, jan-mar/2006, p. 78-104. Disponível em:

$<$ http://www.unisantos.br/mestrado/gestao/egesta/artigos/56.pdf > . Acesso em: 18 Set. 2012.

PATAH, L. Estrutura de Gerenciamento de Projetos em Empresas. In: Simpósio de Administração da Produção, Logística e Operações Internacionais, 5, 2002, São Paulo.

Anais... São Paulo:EditoraQuality Digital, 2002. P. 38-44. 
PATAH, L. Alinhamento Estratégico de Estrutura Organizacional de Projetos: Uma análise de múltiplos casos. 2004. 205 f. Dissertação (Mestrado em Engenharia) - Escola Politécnica da Universidade de São Paulo, São Paulo, 2004.

PROJECT MANAGEMENT INSTITUTE. A Guide to the Project Management Body of Knowledge: DritteAusgabe. 3. ed. [s. 1.]: Editora Project Management Inst, 2004. 403 p. PROMON. Gerenciamento de Projetos. Promon Business \& Technology Review. Rio de Janeiro v. MAIO 08, 2008. Disponível em:

$<$ www.promon.com.br/portugues/noticias/...PBTR\%20GE_para\%20web.pdf >. Acesso em: 26 Ago. 2011.

SILVA, T. E.; TOMAÉL, M. I. A Gestão da Informação nas Organizações. Inf. Inf. Londrina, v. 12, n. 2, jul-dez/2007. Disponível em:

$<$ www.uel.br/revistas/uel/index.php/informacao/article/download/1806/1540>. Acesso em: 10 set. 2012.

SOTILlE, M. Gerenciamento de Projetos na Engenharia de Software. PmTech Capacitação em Projetos de Software. 2004. Disponível em:

$<$ http://www.pmtech.com.br/artigos/Gerenciamento_Projetos_Software.pdf $>$. Acesso em: 26 Ago. 2011.

VARGAS, R. V. Gerenciamento de Projetos: Estabelecendo diferenciais competitivos. 6 . Ed., Rio de Janeiro: Brasport, 2005. 250 p.

VERGARA, S. C. Projetos e Relatórios de Pesquisa em Administração. 6. ed. São Paulo: Editora Atlas, 2005. 102 p.

VIEIRA, E. N. O. Gerenciando Projetos na Era de Grandes Mudanças: Uma breve abordagem do panorama atual. Disponível em:

$<$ http://www.fapparnaiba.com.br/moodle/data/86/artigos/Gerenciando_Projetos_em_na_Era_ de_Grandes_Mudancas.pdf $>$. Acesso em: 24 Ago. 2011. 